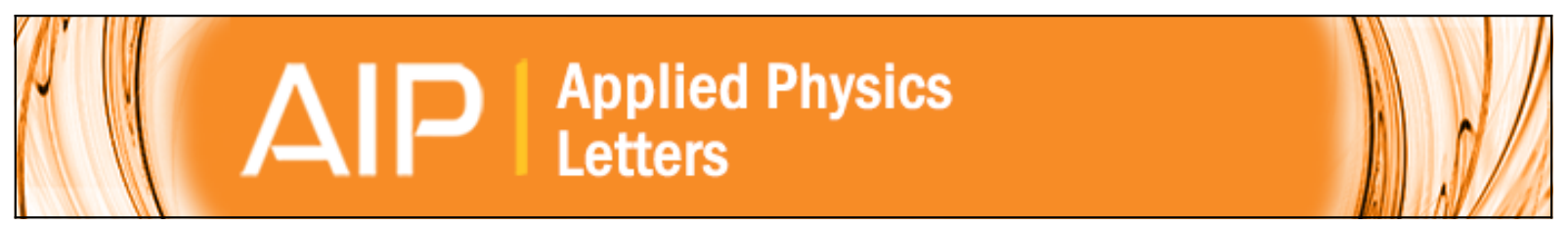

\title{
Short range polar state transitions and deviation from Rayleigh-type behaviour in Bi0.5Na0.5TiO3-based perovskites
}

Giuseppe Viola, Yongqiang Tan, Ruth Agnes McKinnon, Xiaoyong Wei, Haixue Yan, and Michael John Reece

Citation: Applied Physics Letters 105, 102906 (2014); doi: 10.1063/1.4895559

View online: http://dx.doi.org/10.1063/1.4895559

View Table of Contents: http://scitation.aip.org/content/aip/journal/apl/105/10?ver=pdfcov

Published by the AIP Publishing

\section{Articles you may be interested in}

Tuning of dielectric, pyroelectric and ferroelectric properties of $0.715 \mathrm{Bi} 0.5 \mathrm{Na} 0.5 \mathrm{TiO}-0.065 \mathrm{BaTiO}-0.22 \mathrm{SrTiO} 3$ ceramic by internal clamping

AlP Advances 5, 087145 (2015); 10.1063/1.4929328

Phase characteristics of $0.92 \mathrm{Bi} 0.5 \mathrm{Na} 0.5 \mathrm{TiO} 3-0.08 \mathrm{BiAlO} 3$ ceramics

Appl. Phys. Lett. 106, 092903 (2015); 10.1063/1.4913972

Large strain response based on relaxor-antiferroelectric coherence in

Bi0.5Na0.5TiO3-SrTiO3-(K0.5Na0.5) NbO3 solid solutions

J. Appl. Phys. 116, 184104 (2014); 10.1063/1.4901549

Electric-field-temperature phase diagram of the ferroelectric relaxor system (1-x)Bi1/2Na1/2TiO3-xBaTiO3 doped with manganese

J. Appl. Phys. 115, 194104 (2014); 10.1063/1.4876746

Piezoresponse and ferroelectric properties of lead-free [ $\mathrm{Bi} 0.5$ ( $\mathrm{Na} 0.7 \mathrm{~K} 0.2 \mathrm{Li} 0.1$ ) 0.5 ] Ti O 3 thin films by pulsed laser deposition

Appl. Phys. Lett. 92, 222909 (2008); 10.1063/1.2938364

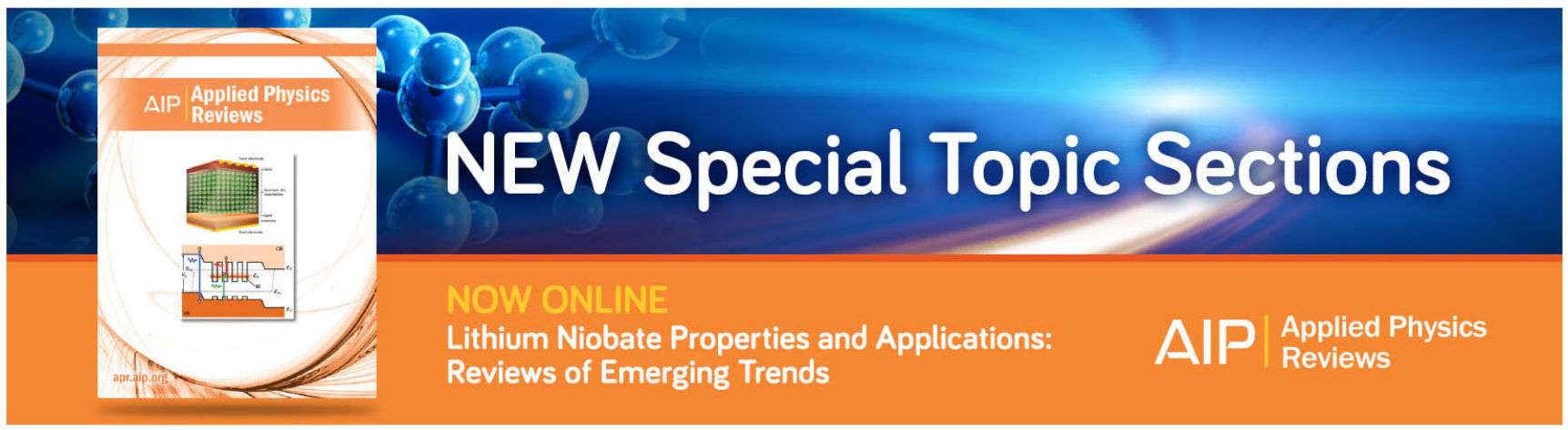




\title{
Short range polar state transitions and deviation from Rayleigh-type behaviour in $\mathrm{Bi}_{0.5} \mathrm{Na}_{0.5} \mathrm{TiO}_{3}$-based perovskites
}

\author{
Giuseppe Viola, ${ }^{1,2}$ Yongqiang Tan, ${ }^{1}$ Ruth Agnes McKinnon, ${ }^{1}$ Xiaoyong Wei, ${ }^{3}$ Haixue Yan, ${ }^{1}$ \\ and Michael John Reece ${ }^{1}$ \\ ${ }_{1}^{1}$ Materials Research Institute, Queen Mary University of London, Mile End Road, London E1 4NS, \\ United Kingdom \\ ${ }^{2}$ Department of Applied Science and Technology, Institute of Materials Physics and Engineering, \\ Corso Duca degli Abruzzi 24, 10129 Torino, Italy \\ ${ }^{3}$ Electronic Materials Research Laboratory, Key Laboratory of the Ministry of Education and International \\ Center for Dielectric Research, Xian Jiaotong University, Xian 710049, People's Republic of China
}

(Received 7 July 2014; accepted 30 August 2014; published online 12 September 2014)

\begin{abstract}
The polarization response of $0.95\left[0.94\left(\mathrm{Bi}_{0.5} \mathrm{Na}_{(0.45)} \mathrm{Li}_{0.05}\right) \mathrm{TiO}_{3}-0.06 \mathrm{BaTiO}_{3}\right]-0.05 \mathrm{CaTiO}_{3}$ ceramics was studied under weak applied cyclic electric fields with different amplitudes and frequency. The analysis of the polarization signals showed that in the ferroelectric phase the non-linearity is dominated by a Rayleigh-type dynamics, while in the ergodic relaxor phase the polarization response deviates from the Rayleigh-type behaviour due to the occurrence of short range electric field-induced transitions, evidenced by the presence of four distinct frequency independent current broad peaks in the current-electric field loops, which gives rise to a characteristic non-linear polarization-electric field loop with reduced hysteresis and weak frequency dependence. (C) 2014 AIP Publishing LLC.

[http://dx.doi.org/10.1063/1.4895559]
\end{abstract}

Disordered systems such as dipolar glasses, relaxors, and ferroelectrics exhibit a non-linear and frequencydispersive dielectric response when subjected to an external applied electric field. ${ }^{1-3}$ This is caused by different underlying mechanisms, including: (a) the reorientation of polar regions according to the "superparaelectric model,"4 (b) the enlargement of polar nanoregions as described in the so called "breathing model,"5,6 and (c) the interaction of domain walls with pinning points which is often manifested as a Rayleigh-like behaviour. ${ }^{7-11}$ The "superparaelectric model" assumes that local polarization vectors are reoriented via thermally- and field-induced flipping with distributed energy barriers which give rise to the amplitude and frequency dependence of the permittivity. ${ }^{4}$ In the "breathing model,", $, 5,6$ it is imagined that polar regions are embedded in a non-polar matrix and that they enlarge their size through the movement of their walls under the action of the applied field. It is assumed that the walls are partially pinned, hence only a portion of their length can freely move under the action of the electric field. The magnitude of such characteristic length, within which the domain walls can bow, varies due to a random distribution of pinning centres, leading to the amplitude and frequency-dependence of the dielectric response. ${ }^{6}$ Another typical manifestation of non-linearity is represented by the Rayleigh-like behaviour found in different types of ferroic materials under weak cyclic applied fields. ${ }^{7-14}$ The parabolic relationship known as the Rayleigh law was first applied in ferromagnetic materials, where hysteretic magnetisation-magnetic field (M-H) loops were observed under weak applied magnetic fields. ${ }^{7,8}$ Rayleigh-type behaviour was then observed in ferroelectric/ferroelastic systems that display non-linear and hysteretic polarization-electric field $(\mathrm{P}-\mathrm{E})^{9-13}$ and strain-stress $(\mathrm{S}-\sigma)$ loops. ${ }^{14}$ Until recently, it has been commonly accepted that the Rayleigh-type behaviour can be described by the motion of single non-interacting domain walls in a potential energy landscape that is perturbed by the presence of randomly distributed pinning sites. ${ }^{8,9}$ In this framework, the susceptibility has a logarithmic-type dependence on the frequency which can be derived from random field theory. ${ }^{15}$ However, recent investigations on lead zirconate titanate (PZT) thin and thick films demonstrated that the underlying mechanisms of the Rayleigh-type response are based on the motion of interacting domain walls rather than single non-interacting walls. ${ }^{16}$ The non-linearity has been attributed to the dynamic coupling that exists between domain walls in the ferroelectric phase. ${ }^{16}$ In addition, it has been shown that in PZT thin films the Rayleigh-like behaviour observed at the macroscale represents an average of individual nonRayleigh type response probed at the nanoscale originating from the motion of ferroelastic walls. ${ }^{17}$ It is generally accepted that a Rayleigh-type analysis performed by varying the amplitude and frequency of the applied field could estimate the contributions of reversible and irreversible domain walls displacements to the non-linear response of different ferroic systems. ${ }^{8-14}$

In this work, the dielectric non-linearity of bismuthbased perovskites was studied by performing a Rayleightype analysis on the compound $0.95\left[0.94\left(\mathrm{Bi}_{0.5} \mathrm{Na}_{(0.45)} \mathrm{Li}_{0.05}\right)\right.$ $\left.\mathrm{TiO}_{3}-0.06 \mathrm{BaTiO}_{3}\right]-0.05 \mathrm{CaTiO}_{3}$, in the temperature range $23^{\circ} \mathrm{C}-150{ }^{\circ} \mathrm{C}$. This particular system previously characterized ${ }^{18}$ was chosen because it experiences a transition from a ferroelectric polar phase to a weakly polar relaxor state with increasing temperature, and a transition from weakly polar to ferroelectric state, with a change in the crystal structure, during the application of an electric field. ${ }^{18}$ The harmonic analysis of the Rayleigh-type response in such system could potentially provide additional understanding on the possible interconnections between electric field-induced transitions and dielectric non-linearity in $\mathrm{Bi}_{0.5} \mathrm{Na}_{0.5} \mathrm{TiO}_{3}$ (BNT)-based 
ceramics and in other systems that experience electric-field induced transitions.

The experiments were carried out on an initially unpoled specimen, using a ferroelectric hysteresis measurement tester (National Physical Laboratory, UK) ${ }^{19,20}$ at different temperatures in the range $23^{\circ} \mathrm{C}-150^{\circ} \mathrm{C}$. Currentpolarization-electric field (I-P-E) loops were generated using sine waveforms of different electric field amplitudes in the range $2.5-17.5 \mathrm{kV} / \mathrm{cm}$ at five different frequencies 1 , $3,5,7$, and $10 \mathrm{~Hz}$. The electric field amplitudes used are significantly lower than the electric field thresholds of the forward transitions $\pm \mathrm{E}_{\mathrm{F}}$ previously identified from the presence of current peaks during cycling at higher electric field amplitudes $(60-70 \mathrm{kV} / \mathrm{cm}) .{ }^{18}$ In the range $100^{\circ} \mathrm{C}-125^{\circ} \mathrm{C}$, the applied amplitudes are comparable with the electric fields labelled as the backward transition thresholds $\pm \mathrm{E}_{\mathrm{B}}$. However, the latter correspond to current peaks which appear only at electric field regime cycling conditions when the electric field is increased beyond the threshold $\pm \mathrm{E}_{\mathrm{F}},{ }^{18}$ and therefore are not considered in the present discussion.
Figure 1 shows the current-polarization-electric field curves generated at $5.0 \mathrm{kV} / \mathrm{cm}$ (Fig. 1(a)) and $17.5 \mathrm{kV} / \mathrm{cm}$ (Fig. $1(\mathrm{~b}))$ at $5 \mathrm{~Hz}$ in the range $23^{\circ} \mathrm{C}-150^{\circ} \mathrm{C}$. It can be seen that the hysteresis of the P-E loops decreases with increasing temperature and the P-E loop changes shape. With increasing electric field amplitude and temperature, the I-E loops start to change from an elliptic shape to a hippopede-like shape, which become clearer and more distinct at $17.5 \mathrm{kV} / \mathrm{cm}$ amplitude and $150{ }^{\circ} \mathrm{C}$ (Fig. 1(b)) with the presence of broad peaks separated by a depression in the electric field interval around $\mathrm{E}=0$, related to the constriction that occurs in the centre region at near zero field of the P-E loops. In Figure 1, it is noted that in the P-E loops generated under the same electric field amplitude and frequency, the maximum polarisation increases with increasing temperature until $125^{\circ} \mathrm{C}$ $\left(\mathrm{P}_{\max }=0.0734 \mathrm{C} / \mathrm{m}^{2}\right.$ at $17.5 \mathrm{kV} / \mathrm{cm}$ and $\left.5 \mathrm{~Hz}\right)$ and then it starts to decrease $\left(\mathrm{P}_{\max }=0.0721 \mathrm{C} / \mathrm{m}^{2}\right.$ at $150{ }^{\circ} \mathrm{C}, 17.5 \mathrm{kV} /$ $\mathrm{cm}$, and $5 \mathrm{~Hz}$ ). A similar effect was also previously observed in the P-E loops of $0.95\left[0.94\left(\mathrm{Bi}_{0.5} \mathrm{Na}_{0.5}\right) \mathrm{TiO}_{3}-0.06 \mathrm{BaTiO}_{3}\right]-$ $0.05 \mathrm{CaTiO}_{3}$ generated at higher electric field amplitudes and (a)

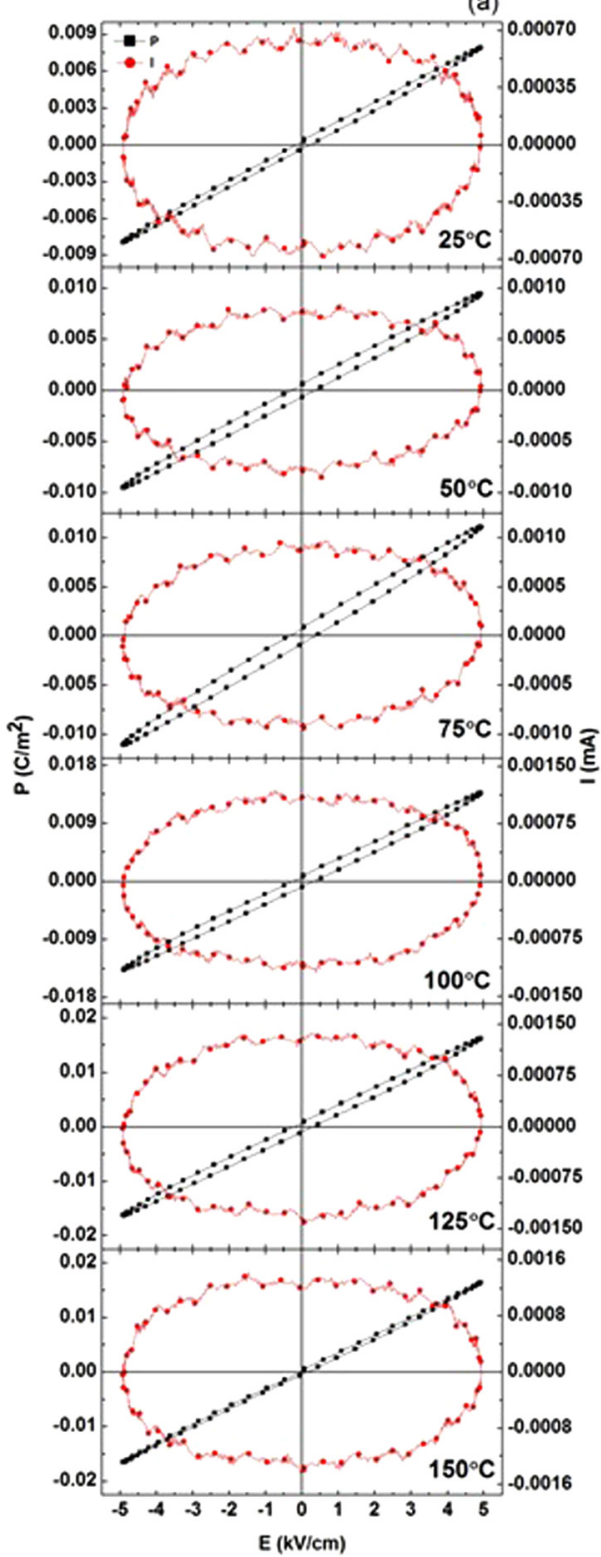

(b)

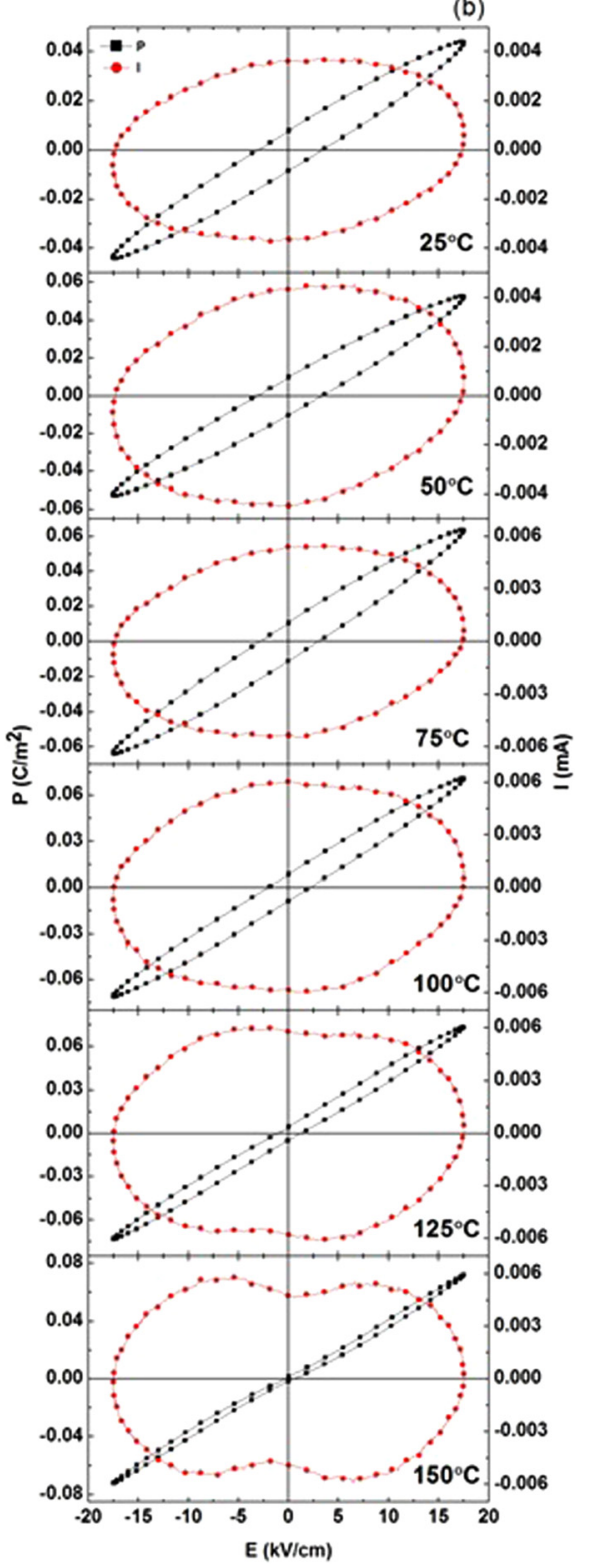

FIG. 1. Current-polarization-electric field loops at different temperature in the range $23{ }^{\circ} \mathrm{C}-150{ }^{\circ} \mathrm{C}$ generated at $5 \mathrm{kV} / \mathrm{cm}$, $5 \mathrm{~Hz}$ (Fig. 1(a)) and $17.5 \mathrm{kV} / \mathrm{cm}, 5 \mathrm{~Hz}$ (Fig. 1(b)). 
it was proposed that it could be related to an electric fieldinduced transformation from a weakly polar to polar state. ${ }^{21}$ The presence of this effect also in the low amplitude regime here reported, suggests that the transition from weakly polar to polar order starts in the low field range, and it culminates at the electric field corresponding to the sharp current peaks observed in the loops generated at higher electric field amplitude (at $\pm \mathrm{E}_{\mathrm{F}}$ as reported in Ref. 18).

Figure 2 shows that the position of the current broad peaks in the loops generated at $17.5 \mathrm{kV} / \mathrm{cm}$ and $150^{\circ} \mathrm{C}$ is independent from the frequency of the applied field. The I-E loops at $3,5,7$, and $10 \mathrm{~Hz}$ have been plotted using the left $\mathrm{y}$-axis, while the I-E loop at $1 \mathrm{~Hz}$ is relative to the right y-axis (Fig. 2). It can be seen that the I-E loops at $1 \mathrm{~Hz}$ and $10 \mathrm{~Hz}$ have practically the same shape and the current broad peaks overlap exactly.

Based on in-situ X-ray diffraction experiments, Jo et al. proposed that in $0.94 \mathrm{Bi}_{0.5} \mathrm{Na}_{0.5} \mathrm{TiO}_{3}-0.06 \mathrm{BaTiO}_{3}$ the electric field-induced transition takes place in two main steps represented by the alignment of randomly oriented polar nanoregions or nanodomains along the external field direction (step I), and then by the enlargement of those with the consequent establishment of a long-range ferroelectric order upon a further increase of the applied field (step II). ${ }^{22}$ Therefore, the current broad peaks observed in the I-E loops during electric field loading and unloading (Fig. 1(b)) could be related to the step I of the electric field-induced transition: ${ }^{22}$ during electrical loading in the weak field range, polar nanoregions are forced to develop a short range polar order (at $\pm E_{\mathrm{SRF}}$ in Fig. 2), while when the electric field is removed most of the polar regions recover their original configuration (at $\pm \mathrm{E}_{\mathrm{SRB}}$ in Fig. 2). Current broad peaks can be also spotted in the low electric field regime of the I-E loops at $200^{\circ} \mathrm{C}$ of $\mathrm{Bi}_{0.5} \mathrm{Na}_{0.5} \mathrm{TiO}_{3}-\mathrm{BaTiO}_{3}$ doped with manganese, ${ }^{23}$ suggesting that step I is common to several the BNT-based ceramics, but the correspondent current broad peaks are probably observable only when they do not get swamped in the background of the current signal.

The constancy of the shape and the position of the current broad peaks in the I-E loops generated at different frequency (Fig. 2) suggests that the underlying process is not thermally assisted within the amplitude and frequency range investigated, but it is triggered once the electric field

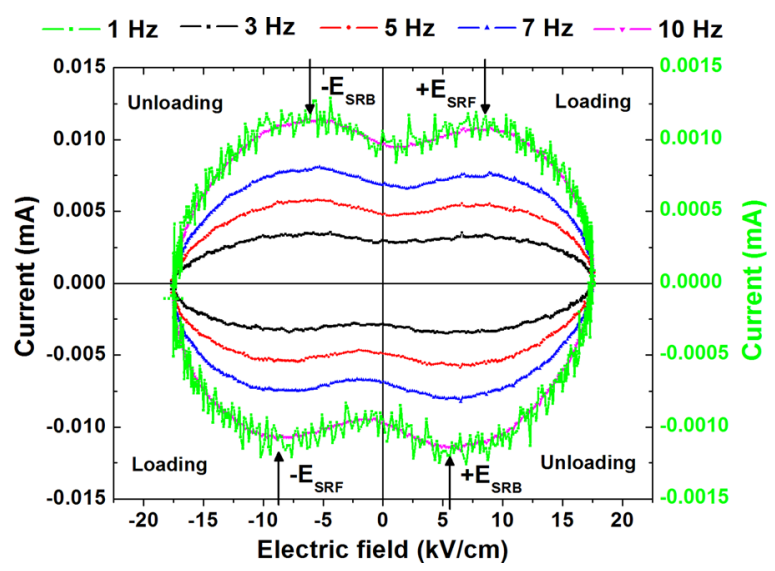

FIG. 2. I-E loops at $17.5 \mathrm{kV} / \mathrm{cm}$ and different frequencies at $150^{\circ} \mathrm{C}$. reaches a critical value (note the absence of current broad peaks at $150^{\circ} \mathrm{C}$ under $5 \mathrm{kV} / \mathrm{cm}$ amplitude in Fig. 1(a)), regardless of the frequency at which it is applied. The independence on the frequency may also originate from the recoverable character of the underlying mechanism, which is also responsible for the reduced hysteresis of the P-E loops with increasing temperature (Fig. 1). Reversible processes are anhysteretic and rate independent (i.e., elastic deformations do not present dependence on the rate of the applied stress). The underlying process is, however, not entirely recoverable, as proved by the following experimental test. A virgin sample was heated at $150^{\circ} \mathrm{C}$ and it was subjected to an electric field waveform with $17.5 \mathrm{kV} / \mathrm{cm}$ amplitude and $5 \mathrm{~Hz}$ frequency; after the test the sample was cooled at room temperature where it showed a $\mathrm{d}_{33}=1.1$ $\mathrm{pC} / \mathrm{N}$, meaning that a tiny residual polar state remained after removing the electrical loading. This could be responsible for the small hysteresis observed in the P-E loop at $\mathrm{T}=150^{\circ} \mathrm{C}$ (Fig. 1(b)).

As commonly done in Rayleigh-type analysis, the relative permittivity $\varepsilon$ was estimated from the slope of the P-E loops by linear fitting and plotted as a function of the electric field amplitude at different frequencies in the temperature range $23^{\circ} \mathrm{C}-150{ }^{\circ} \mathrm{C}$ (see Fig. 3). A close match with the permittivity values obtained from the slope of the P-E loops at $2.5 \mathrm{kV} / \mathrm{cm}$ electric field amplitude and those previously obtained from the impedance spectroscopy measurements in the permittivity-temperature $\operatorname{plot}^{18}$ has been verified. As the electric field amplitude increases, the estimated permittivity increases because a greater extrinsic contribution from domain wall movement is produced in the ferroelectric phase, ${ }^{9,10}$ and a larger extent of the short range transition is induced in the weakly polar relaxor phase. Interestingly, the frequency dispersion of the permittivity at $150{ }^{\circ} \mathrm{C}$ is significantly smaller compared with that observed at lower temperatures (Fig. 3).

In the case of ideal Rayleigh behaviour, the permittivity increases linearly with increasing electric field amplitude ${ }^{9,10}$
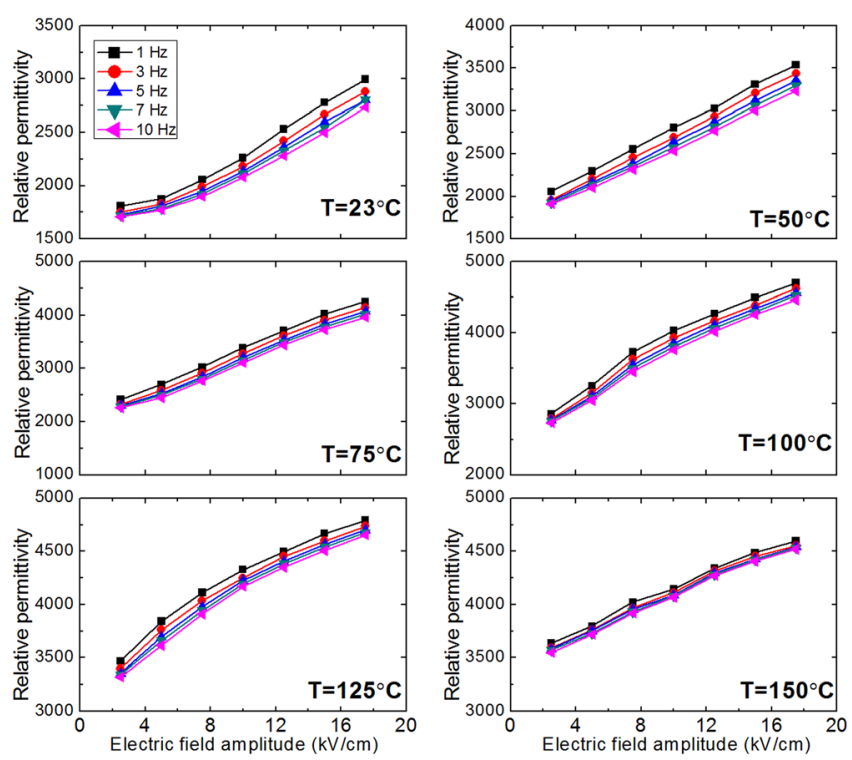

FIG. 3. Dielectric permittivity-electric field amplitude at different frequencies in the range $23^{\circ} \mathrm{C}-150{ }^{\circ} \mathrm{C}$. 


$$
\varepsilon=\varepsilon_{\text {init }}+\alpha \Delta E
$$

where $\varepsilon_{\text {init }}$ is the dielectric permittivity at zero electric field amplitude $\Delta E$, and $\alpha$ is a coefficient which describes the degree of non-linearity of the P-E loop, such that the polarization response as a function of the electric field $E$ during an electric field cycle of amplitude $\Delta E$ can be written in terms of the well-known Rayleigh law ${ }^{9,10}$

$$
P(E)=\left(\varepsilon_{\text {init }}+\alpha \Delta E\right) E \pm \frac{\alpha}{2}\left(\Delta E^{2}-E^{2}\right) .
$$

The harmonic analysis of the polarization response generally allows for a more accurate evaluation of the deviation from the ideal Rayleigh behaviour compared to a mere polynomial fitting of the P-E loops and it may provide further information on the underlying mechanisms of the non-linear and hysteretic behaviour in the low electric field amplitude regime. For this purpose, a harmonic analysis of the polarization (P)-time (t) signals was performed by spectral decomposition into a Fourier series of harmonic functions. The polarization signals were expressed as the sum of $n$ harmonics

$$
\begin{aligned}
P(t)= & P_{1}^{\prime} \sin (\omega t)+P_{1}^{\prime \prime} \cos (\omega t)+P_{2}^{\prime} \sin (2 \omega t) \\
& +P_{2}^{\prime \prime} \cos (2 \omega t)+P_{3}^{\prime} \sin (3 \omega t)+P_{3}^{\prime \prime} \cos (3 \omega t) \\
& +\cdots+P_{n}^{\prime} \sin (n \omega t)+P_{n}^{\prime \prime} \cos (n \omega t) \\
= & \sum_{n=1,2 \ldots}^{\infty} \sqrt{\left(P_{n}^{\prime}\right)^{2}+\left(P_{n}^{\prime \prime}\right)^{2}} \sin \left(n \omega t+\delta_{n}\right),
\end{aligned}
$$

where $\omega$ is the angular frequency, $P_{n}^{\prime}$ and $P_{n}^{\prime \prime}$ are the in-phase and out-of-phase amplitudes, and $\delta_{n}=\arctan \left(\frac{P_{n}^{\prime \prime}}{P_{\prime}^{\prime}}\right)$ is the phase angle of the $n$th harmonic. ${ }^{9,24-26}$ The number of harmonics, together with their relative amplitudes and phase angles were determined by a best fit procedure of the polarization signals using Matlab software. The amplitudes and phase angles of all the harmonics were studied as a function of the electric field amplitude in the loops generated at $5 \mathrm{~Hz}$ at different temperatures in the range $23^{\circ} \mathrm{C}-150^{\circ} \mathrm{C}$. The non-linear behaviour can be still classified as ideal Rayleightype, even when the higher order harmonics are present, as long as there are only odd harmonics and their phase angles are equal to $\pm 90^{\circ}$. This implies that the non-linear response given by the high order harmonics is also hysteretic. ${ }^{9,24-26}$

The coefficients of the Fourier series expansion were tabulated as a function of electric field amplitude and temperature. For brevity, while still allowing tracking the main trends in the parameters of the harmonics, only the values relative to the analysis performed on the polarization signals obtained at $2.5 \mathrm{kV} / \mathrm{cm}, 10 \mathrm{kV} / \mathrm{cm}$, and $17.5 \mathrm{kV} / \mathrm{cm}$ amplitude and $5 \mathrm{~Hz}$ frequency are reported in Tables 1-3, respectively, given in the supplementary material. ${ }^{27}$ In virtue of the so called half wave symmetry of the $\mathrm{P}=\mathrm{P}(\mathrm{t})$ signal (which verifies the property $P(\omega t)=-P(\omega t+\pi)),{ }^{24}$ the Fourier series in all of the $\mathrm{P}=\mathrm{P}(\mathrm{t})$ signals reported in Tables $1-3^{27}$ present only odd harmonics, inferring the absence of any biased polarization state as confirmed by $P(\Delta E)=-P(-\Delta E)$ in the loops generated (see Fig. 1). The results of the analysis show that the number of odd harmonics generally increases with increasing electric field amplitude and temperature. In the polarization signals relative to the loops generated at $2.5 \mathrm{kV} / \mathrm{cm}$ amplitude, only the first order harmonic is present in the entire temperature range (Table 1$).^{27}$ At $10 \mathrm{kV} / \mathrm{cm}$ amplitude, a larger number of harmonics was needed to fit the polarization signals (Table 2), ${ }^{27}$ which further increased in the case of $17.5 \mathrm{kV} / \mathrm{cm}$ amplitude (Table 3$){ }^{27}$ The coefficients of the high order harmonics are often too small to provide accurate phase angles to identify the onset of the departure from the ideal Rayleigh behaviour. This is also due to tiny deviations of the applied alternating electric field from the pure sine waveform, which were detected from a harmonic analysis performed on the signal $E=E(t)$. We observed that the phase angles of the high order harmonics are not sufficiently reliable when the parameters $\mathrm{P}^{\prime}$ and $\mathrm{P}^{\prime \prime}$ of the high order harmonics are smaller than $\pm 0.0005 \mathrm{C} / \mathrm{m}^{2}$ (see values in italic font in the Tables ${ }^{27}$ ). Since from the phase angles of the high order harmonics, it was hard to identify the electric field amplitude and temperature where the material starts to deviate from the ideal Rayleigh response, the deviations were also examined by fitting the P-E loops with the Rayleigh law in Eq. (2) (plots not shown here). It was concluded that until $75^{\circ} \mathrm{C}$ the non-linear behaviour is dominated by a Rayleigh-type response in the entire range of electric field amplitudes considered. As the temperature is increased, the behaviour increasingly deviates from the Rayleigh-type and at $150{ }^{\circ} \mathrm{C}$ under $17.5 \mathrm{kV} / \mathrm{cm}$ amplitude, it seems clear that the material does not follow a Rayleigh-type dynamics, showing constricted polarizationelectric field loops with reduced hysteresis. The parameters $\mathrm{P}_{3}{ }^{\prime}$ and $\mathrm{P}_{5}^{\prime}$ at $150{ }^{\circ} \mathrm{C}$ are both bigger than 0.0005 (Table 3 supplemental material ${ }^{27}$ ) which ensures that the phase angles of the third and fifth harmonics are definitely different from $\pm 90^{\circ}$, confirming the presence of a non-Rayleigh regime. The constricted P-E loop observed at $150{ }^{\circ} \mathrm{C}$ (see Fig. 1) can be associated with the progressive stability of a weakly polar state with increasing temperature in absence of an applied electric field (remnant polarization tending to zero), well documented in bismuth-based perovskites. ${ }^{28,29}$

The temperature dependence of the coefficients in the first harmonic is plotted in Fig. 4 from $7.5 \mathrm{kV} / \mathrm{cm}$ onwards, where all the coefficients are higher than \pm 0.0005 . It can be seen that the coefficient $\mathrm{P}_{1}{ }^{\prime}$ shows a peak at $125^{\circ} \mathrm{C}$ at all the amplitudes considered (Fig. 4(a)). This corresponds to the transition temperature $\mathrm{T}_{\mathrm{F}-\mathrm{R}}$ from the ferroelectric to the ergodic relaxor weakly polar phase since it coincides with the temperature where the derivative of the permittivity with respect to temperature shows a maximum (see definition of $\mathrm{T}_{\mathrm{F}-\mathrm{R}}$ in Ref. 22). This was obtained from the permittivity data previously published ${ }^{18}$ and the plots are not shown here. The $\mathrm{P}_{1}{ }^{\prime \prime}$ and $\delta_{1}$ (Figs. 4(b) and 4(c)) values are related to the hysteresis area of the P-E loops. Both parameters increase with increasing electric field amplitude as expected. Their temperature dependence is characterized by an initial increase and by a subsequent decrease after a peak with increasing temperature, consistent with the temperature dependence of the dielectric loss previously reported. ${ }^{18}$

Since the current broad peaks can be attributed to the establishment of an electric field-induced short range polar state (step I), the sharper current peaks found at $\pm \mathrm{E}_{\mathrm{F}}{ }^{18}$ should therefore correspond to the formation of a long range 

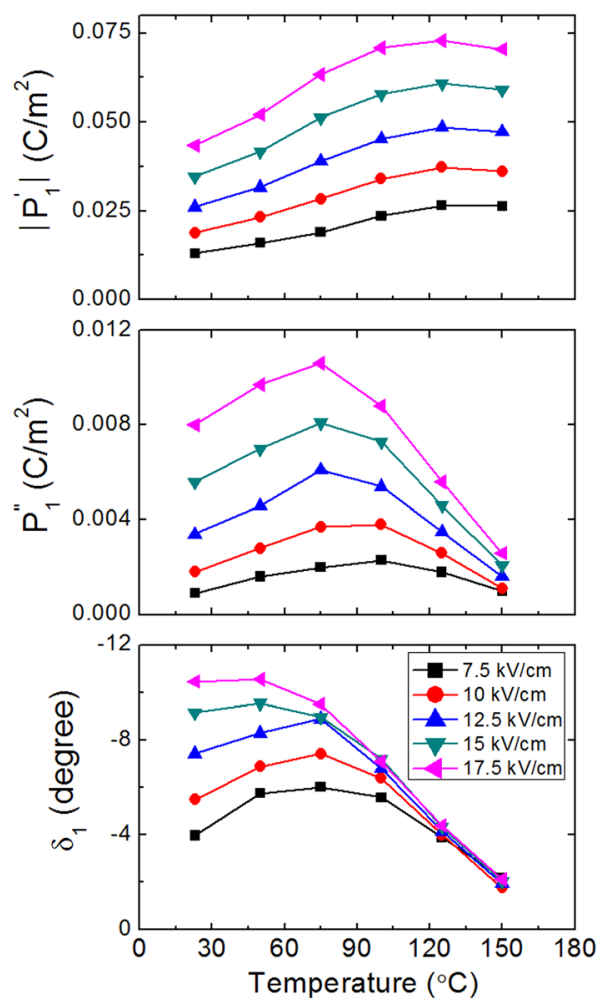

FIG. 4. Temperature dependence of the first harmonic parameters at different electric field amplitude and $5 \mathrm{~Hz}$ frequency.

polar order by the growth and percolation of polar regions (locally induced during step I) through the movement of their boundaries (step II), which is a thermally activated process. This would justify the frequency dispersion of the characteristic electric field thresholds previously reported in other BNT-based systems. ${ }^{21,30}$ Percolation of polar nanoregions during electrical loading were also recently found in barium zirconate titanate, ${ }^{31}$ suggesting that the mechanisms of electric field induced transitions in different relaxor-like materials share similar features.

In conclusion, the non-linear behaviour in the ferroelectric phase of $0.95\left[0.94\left(\mathrm{Bi}_{0.5} \mathrm{Na}_{(0.45)} \mathrm{Li}_{0.05}\right) \mathrm{TiO}_{3}-0.06 \mathrm{BaTiO}_{3}\right]-$ $0.05 \mathrm{CaTiO}_{3}$ is dominated by Rayleigh-like features. The non-linear behaviour increasingly deviates from the Rayleightype response with increasing temperature. In the weakly polar relaxor state, the characteristic non-linear polarizationelectric field response is attributed to the occurrence of an electric field-induced transition which starts taking place in the low electric field amplitude regime, with the establishment of a short range polar state. The process is mainly recoverable as indicated by a remnant polarization close to zero and a narrow P-E loop hysteresis loop, and it is not thermally activated as suggested by the absence of frequency dispersion in current-polarization-electric field loops within the range of electric field amplitudes and frequencies studied. It is expected that the described mechanisms are common in different $\mathrm{Bi}_{0.5} \mathrm{Na}_{0.5} \mathrm{TiO}_{3}$-based perovskites and in other systems experiencing electric-field induced transitions.

${ }^{1}$ K. B. Lyons, P. A. Fleury, T. J. Negran, and H. L. Carter, Phys. Rev. B 36, 2465 (1987).

${ }^{2}$ A. E. Glazounov and A. K. Tagantsev, Phys. Rev. Lett. 85(10), 2192 (2000).

${ }^{3}$ A. E. Glazounov and A. K. Tagantsev, J. Phys.: Condens. Matter 10, 8863 (1998).

${ }^{4}$ A. J. Bell, J. Phys.: Condens. Matter 5, 8773 (1993).

${ }^{5}$ A. E. Glazounov, A. K. Tagantsev, and A. J. Bell, Phys. Rev. B 53, 11281 (1996).

${ }^{6}$ A. E. Glazounov and A. K. Tagantsev, Ferroelectrics 221, 57 (1999).

${ }^{7}$ L. Rayleigh, Philos. Mag. 23, 225 (1887).

${ }^{8}$ L. Neel, Cah. Phys. 12, 1 (1942).

${ }^{9}$ D. Damjanovic, "Hysteresis in piezoelectric and ferroelectric materials," in The Science of Hysteresis, edited by I. Mayergoyz and G. Bertotti (Elsevier, 2005), Vol. 3.

${ }^{10}$ D. A. Hall, J. Mater. Sci. 36, 4575 (2001).

${ }^{11}$ D. Damjanovic and M. Demartin, J. Phys. D: Appl. Phys. 29, 2057 (1996).

${ }^{12}$ A. Pramanick, D. Damjanovic, J. C. Nino, and J. L. Jones, J. Am. Ceram. Soc. 92(10), 2291 (2009).

${ }^{13}$ A. Pramanick, J. E. Daniels, and J. L. Jones, J. Am. Ceram. Soc. 92(10), 2300 (2009).

${ }^{14}$ G. Viola, D. Verbylo, N. Orlovskaya, and M. J. Reece, Mater. Sci. Technol. 25(11), 1312 (2009).

${ }^{15}$ T. Nattermann, Y. Shapir, and I. Vilfan, Phys. Rev. B 42(13), 8577 (1990).

${ }^{16} \mathrm{P}$. Bintachitta, S. Jesse, D. Damjanovic, Y. Han, I. M. Reaney, S. TrolierMcKinstry, and S. V. Kalinin, Proc. Natl. Acad. Sci. U. S. A. 107(16), 7219 (2010).

${ }^{17}$ R. K. Vasudevan, M. B. Okatan, C. Duan, Y. Ehara, H. Funakubo, A. Kumar, S. Jesse, L.-Q. Chen, S. V. Kalinin, and V. Nagarajan, Adv. Funct. Mater. 23, 81 (2013).

${ }^{18}$ G. Viola, R. McKinnon, V. Koval, A. Adomkevicius, S. Dunn, and H. Yan, J. Phys. Chem. C 118(16), 8564 (2014).

${ }^{19}$ H. Yan, F. Inam, G. Viola, H. Ning, H. Zhang, Q. Jiang, T. Zhang, Z. Gao, and M. J. Reece, J. Adv. Dielectr. 1(1), 107 (2011).

${ }^{20}$ G. Viola, T. Saunders, X. Wei, K. B. Chong, H. Luo, M. J. Reece, and H. Yan, J. Adv. Dielectr. 3(1), 1350007 (2013).

${ }^{21}$ G. Viola, H. Ning, X. Wei, M. Deluca, A. Adomkevicius, J. Khaliq, M. J. Reece, and H. Yan, J. Appl. Phys. 114, 014107 (2013).

${ }^{22}$ W. Jo, J. Daniels, D. Damjanovic, W. Kleemann, and J. Rodel, Appl. Phys. Lett. 102, 192903 (2013).

${ }^{23}$ E. Sapper, N. Novak, W. Jo, T. Granzow, and J. Rodel, J. Appl. Phys. 115, 194104 (2014).

${ }^{24}$ M. I. Morozov, "Softening and hardening transitions in ferroelectric $\mathrm{Pb}(\mathrm{Zr}, \mathrm{Ti}) \mathrm{O}_{3}$ ceramics," Ph.D. thesis (No. 3368 École Polytechnique Fédérale de Lausanne, 2005).

${ }^{25}$ M. I. Morozov and D. Damjanovic, J. Appl. Phys. 104, 034107 (2008).

${ }^{26}$ D. V. Taylor and D. Damjanovic, Appl. Phys. Lett. 73, 2045 (1998).

${ }^{27}$ See supplementary material at http://dx.doi.org/10.1063/1.4895559 for the coefficients of the Fourier series expansion as a function of electric field amplitude and temperature.

${ }^{28}$ G. O. Jones and P. A. Thomas, Acta Crystallogr., Sect. B: Struct. Sci. 58, 168 (2002).

${ }^{29}$ C. Ma and X. Tan, J. Am. Ceram. Soc. 94(11) 4040 (2011).

${ }^{30}$ R. Dittmer, W. Jo, E. Aulbach, T. Granzow, and J. Rodel, J. Appl. Phys. 112, 014101 (2012).

${ }^{31}$ S. Prosandeev, D. Wang, A. R. Akbarzadeh, B. Dkhil, and L. Bellaiche, Phys. Rev. Lett. 110, 207601 (2013). 